\title{
Integrated Disease Management of Stem and Root Rot of Brinjal caused by Macrophomina phaseolina
}

\author{
A.G. Dapkekar ${ }^{1 *}$, Bholanath Mondal $^{1}$ and A.P. Suryawanshi ${ }^{2}$ \\ ${ }^{1}$ Department of Plant Protection, Palli-Siksha Bhavana (Institute of Agriculture), P.O. \\ Sriniketan, Visva-Bharati, Dist. Birbhum, West Bengal - 731 236, India \\ ${ }^{2}$ Department of Plant Pathology, Dr. BSKKV, Dapoli (M.S), India \\ *Corresponding author
}

\section{Keywords}

Brinjal, $M$. phaseolina, Root rot, Stem rot etc.

Article Info

Accepted:

12 August 2018 Available Online:

10 September 2018

\section{A B S T R A C T}

During the present investigations on charcoal rot caused by Macrophomina phaseolina (Tassi) Goid of Brinjal (Solanum melongena L.), various experiments were conducted at the Department of Plant Pathology, College of Agriculture, Parbhani during 2014-2015 and 2015-16 to fulfill the objective to study the integrated management of stem and root rot disease (M. phaseolina) of brinjal (Pot culture). The experiment was conducted with CRD statistical design and all the treatments were replicated thrice. Overall 14 treatments were evaluated as against $M$. phaseolina (sick soil), sowing susceptible brinjal variety Rakesh in pot culture under screen house conditions. Study shows that all the treatments significantly improved the percentage of seed germination over untreated control (sick soil). On the other hand the percentage reduction in pre-emergence and post-emergence mortalities was ranged from 53.04 to 96.64 per cent and 51.95 to 94.06 per cent, respectively. However, Carbendazim 12\% + Mancozeb 63\% + Trichoderma viride + Neem seed cake $+A$. indica and Carbendazim $50 \mathrm{WP}+T$. harzianum + Cotton seed cake + Z.officinale treatment were found most effective with significantly least pre-emergence seed rot and post emergence seedling mortality.

\section{Introduction}

Brinjal (Solanum melongena L.) is affected by several pathogens including fungi, bacteria, viruses, nematodes and phytoplasmas. The pathogen, Macrophomina phaseolina (Tassi.) Goid is one of the most destructive and wide spread diseases which may cause average losses of 25.60 - $48.62 \%$ (Dinakaran et al., 2005, Jaiman et al., 2009) in many agronomical and horticultural crops. The pathogen being mostly soil borne and polyphagous and hence very difficult to manage with chemicals alone. Therefore, for effective and economical management of the soil borne pathogens like $M$. phaseolina, integrated disease management is need to be explored along with fungicides and other options such as use of biocontrol agents, botanicals and organic amendments.

Therefore, present studies on stem and root rot (M. phaseolina) of brinjal will be undertaken with the objective of studying integrated 
management of stem and root rot disease ( $M$. phaseolina) of brinjal (Pot culture).

\section{Materials and Methods}

Based on the result obtained of the in vitro studies, the best and most effective fungicides, bioagents, botanicals and organic amendments (two each) will be selected and further evaluated (alone and in combination) using earthen pots $(30 \mathrm{~cm}$ dia.) for integrated management of stem/root rot disease $(M$. phaseolina) of brinjal. For the purpose $M$. phaseolina sick soil method will be employed and experiment will be conducted under controlled conditions of the screen house. Any local or improved cultivar of the brinjal most susceptible to $M$. phaseolina will be used. The experiment will be planned with CRD statistical design and all the treatments will be replicated thrice.

\section{Results and Discussion}

A total of 14 treatments were evaluated as against $M$. phaseolina (sick soil), sowing susceptible brinjal variety Rakesh in pot culture (Konde et al., 2008) under screen house conditions. The results obtained on percentage seed germination, pre-emergence seed rot (PESR), post-emergence seedling mortality (PESM) and growth parameters are presented in the Table 1.

\section{Seed germination}

Result revealed that all the treatments significantly improved the percentage seed germination over untreated control (sick soil) and it was ranged from 66.67 to 98.52 per cent as against 28.89 per cent, in untreated control (sick soil). However Carbendazim 12\%+ Mancozeb $63 \%+$ Trichoderma viride + Neem seed cake $+A$. indica treatment was found most effective with significantly highest seed germination $(98.52 \%)$. The second and third best treatments found were Carbendazim 50 $\mathrm{WP}+T$. harzianum + Cotton seed cake $+Z$. officinale $(97.78 \%)$ and Carbendazim $12 \%+$ Mancozeb 63\% (94.08\%), both of which were at par. These were followed by the treatments viz., Carbendazim 50 WP (90.37\%), Thiram $37.5 \%+$ Carboxin $37.5 \%$ (86.67 \%), Trichoderma viride (84.45\%), T. harzianum (82.22 \%), T. hamatum (80.00\%), Neem seed cake $(77.78 \%)$, Cotton seed cake $(75.55 \%)$, Groundnut cake and A. indica (73.33\%), Z.officinale $(68.89 \%)$ and $P$. hystrophorus $(66.67 \%)$.

\section{Pre and post emergence mortalities}

Results revealed that all the treatments significantly influenced both pre-emergence seed rot (PESR) and post emergence seedling mortality (PESM), caused by $M$. phaseolina in brinjal variety Rakesh.

The pre-emergence seed rot (PESR) recorded with all the treatments were ranged from 04.45 to 33.33 per cent, as against 71.11 per cent in untreated control (sick soil).

However, Carbendazim 12\%+ Mancozeb 63\% + Trichodermaviride + Neem seed cake $+A$. indicaand Carbendazim 50 WP $+T$. harzianum + Cotton seed cake + Z.officinale treatments were found most effective with significantly least pre-emergence seed rot 4.45 and 6.67 per cent, respectively and both of which were at par.

These followed by the treatments viz., Carbendazim 12\% + Mancozeb 63\% (8.89\%), Carbendazim 50 WP (11.11\%), Thiram $37.5 \%+$ Carboxin $37.5 \%$ (13.33 \%), Trichoderma viride I (15.55\%), T. harzianum (17.78\%), T. hamatum (20.00\%), Neem seed cake $(22.22 \%)$, Cotton seed cake $(24.45 \%)$, Groundnut cake and A. indica (26.67\%), Z.officinale (31.11 \%), and P.hystrophorus $(33.33 \%)$. 
Table.1 Effect of fungicides, botanicals, bioagents and organic amendments on seed rot and seedling mortality in brinjal (Pot culture)

\begin{tabular}{|c|c|c|c|c|c|c|c|c|c|c|c|}
\hline \multirow[t]{2}{*}{$\begin{array}{l}\text { Tr. } \\
\text { No. }\end{array}$} & \multirow[t]{2}{*}{ Treatments } & \multirow{2}{*}{$\begin{array}{c}\text { Rate (g } \\
\text { or } \mathrm{ml} / \mathrm{kg} \\
\text { seed or } \\
\text { soil) }\end{array}$} & \multirow{2}{*}{$\begin{array}{c}\text { Av. } \\
\text { Germination* } \\
(\%)\end{array}$} & \multirow{2}{*}{$\begin{array}{c}\text { Increase } \\
(\%) \\
\text { over } \\
\text { control }\end{array}$} & \multicolumn{2}{|c|}{$\begin{array}{c}\text { Rot / Mortality } \\
(\%)\end{array}$} & \multirow{2}{*}{$\begin{array}{c}\text { Av. } \\
\text { Mortality } \\
(\%)\end{array}$} & \multicolumn{2}{|c|}{$\begin{array}{c}\text { Reduction }(\%) \\
\text { over control }\end{array}$} & \multirow{2}{*}{$\begin{array}{c}\text { Av. } \\
\text { Reduction } \\
(\%)\end{array}$} & \multirow{2}{*}{$\begin{array}{c}\text { Final } \\
\text { Plant } \\
\text { Stand } \\
(\%)\end{array}$} \\
\hline & & & & & PESR & PESM & & PESR & PESM & & \\
\hline T1 & $\begin{array}{c}\text { Carbendazim } 12 \% \\
+ \text { Mancozeb } 63 \% \\
\text { (ST) }\end{array}$ & $2.0 \mathrm{~g}$ & $\begin{array}{c}94.08 \\
(77.12)\end{array}$ & 69.29 & $\begin{array}{c}08.89 \\
(17.11)\end{array}$ & $\begin{array}{c}09.52 \\
(17.73)\end{array}$ & $\begin{array}{c}08.56 \\
(16.95)\end{array}$ & $\begin{array}{c}90.58 \\
(74.84)\end{array}$ & $\begin{array}{c}87.26 \\
(69.33)\end{array}$ & $\begin{array}{c}90.25 \\
(72.07)\end{array}$ & 82.22 \\
\hline $\mathbf{T}_{2}$ & $\begin{array}{c}\text { Carbendazim } 50 \\
\text { WP (ST) }\end{array}$ & $1.0 \mathrm{~g}$ & $\begin{array}{c}90.37 \\
(72.84)\end{array}$ & 68.03 & $\begin{array}{c}11.11 \\
(19.26)\end{array}$ & $\begin{array}{c}12.45 \\
(20.46)\end{array}$ & $\begin{array}{c}11.41 \\
(19.71)\end{array}$ & $\begin{array}{c}84.55 \\
(67.00)\end{array}$ & $\begin{array}{c}83.79 \\
(66.44)\end{array}$ & $\begin{array}{c}84.17 \\
(66.59)\end{array}$ & 77.78 \\
\hline $\mathbf{T}_{3}$ & $\begin{array}{c}\text { Thiram } 37.5 \%+ \\
\text { Carboxin } 37.5 \% \\
\text { (ST) }\end{array}$ & $2.0 \mathrm{~g}$ & $\begin{array}{c}86.67 \\
(68.56)\end{array}$ & 66.67 & $\begin{array}{c}13.33 \\
(21.41)\end{array}$ & $\begin{array}{c}15.38 \\
(23.08)\end{array}$ & $\begin{array}{c}14.36 \\
(22.26)\end{array}$ & $\begin{array}{c}81.22 \\
(64.30)\end{array}$ & $\begin{array}{c}79.92 \\
(63.36)\end{array}$ & $\begin{array}{c}80.57 \\
(63.82)\end{array}$ & 73.33 \\
\hline $\mathbf{T}_{4}$ & $\begin{array}{l}\text { Trichodermaviride } \\
\qquad(5 \times 106 \mathrm{cfu} / \mathrm{g} \\
\text { carrier) }(\mathrm{ST})\end{array}$ & $8.0 \mathrm{~g}$ & $\begin{array}{c}84.45 \\
(66.84)\end{array}$ & 65.79 & $\begin{array}{c}15.55 \\
(23.12)\end{array}$ & $\begin{array}{c}18.38 \\
(25.29)\end{array}$ & $\begin{array}{c}17.34 \\
(24.58)\end{array}$ & $\begin{array}{c}78.19 \\
(62.22)\end{array}$ & $\begin{array}{c}76.14 \\
(60.79)\end{array}$ & $\begin{array}{c}77.16 \\
(61.46)\end{array}$ & 68.89 \\
\hline $\mathbf{T}_{5}$ & $\begin{array}{l}\text { T. harzianum } \\
\text { (5 X } 106 \mathrm{cfu} / \mathrm{g} \\
\text { carrier) }(\mathrm{ST})\end{array}$ & $8.0 \mathrm{~g}$ & $\begin{array}{c}82.22 \\
(65.13)\end{array}$ & 64.86 & $\begin{array}{c}17.78 \\
(24.84)\end{array}$ & $\begin{array}{c}21.58 \\
(27.59)\end{array}$ & $\begin{array}{c}20.42 \\
(26.84)\end{array}$ & $\begin{array}{c}74.85 \\
(60.00)\end{array}$ & $\begin{array}{c}71.92 \\
(58.04)\end{array}$ & $\begin{array}{c}73.39 \\
(58.95)\end{array}$ & 66.67 \\
\hline $\mathrm{T}_{6}$ & $\begin{array}{l}\text { T. hamatum } \\
\text { (5 X } 106 \mathrm{cfu} / \mathrm{g} \\
\text { carrier) }(\mathrm{ST})\end{array}$ & $8.0 \mathrm{~g}$ & $\begin{array}{c}80.00 \\
(63.41)\end{array}$ & 63.89 & $\begin{array}{c}20.00 \\
(26.55)\end{array}$ & $\begin{array}{c}25.00 \\
(29.99)\end{array}$ & $\begin{array}{c}22.50 \\
(28.31)\end{array}$ & $\begin{array}{c}71.82 \\
(57.92)\end{array}$ & $\begin{array}{c}67.36 \\
(55.14)\end{array}$ & $\begin{array}{c}69.59 \\
(56.51)\end{array}$ & 60.00 \\
\hline$\overline{\mathbf{T}_{7}}$ & $\begin{array}{c}\text { Neem seed cake } \\
(\mathrm{SA})\end{array}$ & $50 \mathrm{~g}$ & $\begin{array}{c}77.78 \\
(61.90)\end{array}$ & 62.86 & $\begin{array}{c}22.22 \\
(28.06)\end{array}$ & $\begin{array}{c}22.73 \\
(28.40)\end{array}$ & $\begin{array}{c}21.73 \\
(27.77)\end{array}$ & $\begin{array}{c}68.79 \\
(56.05)\end{array}$ & $\begin{array}{c}70.39 \\
(57.06)\end{array}$ & $\begin{array}{c}69.59 \\
(56.51)\end{array}$ & 60.00 \\
\hline $\mathbf{T}_{8}$ & $\begin{array}{c}\text { Cotton seed cake } \\
\text { (SA) }\end{array}$ & $50 \mathrm{~g}$ & $\begin{array}{c}75.55 \\
(60.39)\end{array}$ & 61.76 & $\begin{array}{c}24.45 \\
(29.57)\end{array}$ & $\begin{array}{c}26.51 \\
(30.97)\end{array}$ & $\begin{array}{c}25.11 \\
(30.04)\end{array}$ & $\begin{array}{c}65.75 \\
(54.18)\end{array}$ & $\begin{array}{c}65.34 \\
(53.93)\end{array}$ & $\begin{array}{c}65.55 \\
(54.06)\end{array}$ & 55.56 \\
\hline$\overline{T_{9}}$ & $\begin{array}{l}\text { Groundnut cake } \\
\text { (SA) }\end{array}$ & $50 \mathrm{~g}$ & $\begin{array}{c}73.33 \\
(58.88)\end{array}$ & 60.60 & $\begin{array}{c}26.67 \\
(31.08)\end{array}$ & $\begin{array}{c}30.30 \\
(33.34)\end{array}$ & $\begin{array}{c}28.49 \\
(32.23)\end{array}$ & $\begin{array}{c}62.42 \\
(52.18)\end{array}$ & $\begin{array}{c}60.61 \\
(51.13)\end{array}$ & $\begin{array}{c}61.52 \\
(51.65)\end{array}$ & 51.11 \\
\hline $\mathrm{T}_{10}$ & A. indica (SD) & $50 \mathrm{ml}$ & $\begin{array}{c}73.33 \\
(58.88)\end{array}$ & 60.60 & $\begin{array}{c}26.67 \\
(31.08)\end{array}$ & $\begin{array}{c}30.30 \\
(33.34)\end{array}$ & $\begin{array}{c}28.49 \\
(32.23)\end{array}$ & $\begin{array}{c}62.42 \\
(52.18)\end{array}$ & $\begin{array}{c}60.36 \\
(51.00)\end{array}$ & $\begin{array}{c}61.39 \\
(51.58)\end{array}$ & 51.11 \\
\hline$\overline{\mathbf{T}_{11}}$ & Z.officinale (SD) & $50 \mathrm{ml}$ & $\begin{array}{c}68.89 \\
(56.11)\end{array}$ & 58.06 & $\begin{array}{c}31.11 \\
(33.86)\end{array}$ & $\begin{array}{c}29.09 \\
(32.62)\end{array}$ & $\begin{array}{c}30.84 \\
(33.72)\end{array}$ & $\begin{array}{c}56.06 \\
(48.49)\end{array}$ & $\begin{array}{c}62.51 \\
(51.95)\end{array}$ & $\begin{array}{c}56.84 \\
(48.93)\end{array}$ & 48.89 \\
\hline $\mathbf{T}_{12}$ & $\begin{array}{l}\text { P.hystrophorus } \\
\text { (SD) }\end{array}$ & $50 \mathrm{ml}$ & $\begin{array}{c}66.67 \\
(54.72)\end{array}$ & 56.67 & $\begin{array}{c}33.33 \\
(35.25)\end{array}$ & $\begin{array}{c}36.67 \\
(37.21)\end{array}$ & $\begin{array}{c}35.00 \\
(36.25)\end{array}$ & $\begin{array}{c}53.04 \\
(46.72)\end{array}$ & $\begin{array}{c}51.95 \\
(46.12)\end{array}$ & $\begin{array}{c}52.49 \\
(46.41)\end{array}$ & 42.22 \\
\hline$\overline{\mathbf{T}_{13}}$ & $\begin{array}{c}\text { (Carbendazim } \\
\text { 12\%+ Mancozeb } \\
63 \%) \mathrm{ST}+ \\
\text { Trichodermaviride } \\
(\mathrm{ST})+\text { Neem seed } \\
\text { cake (SA) + A. } \\
\text { indica }(\mathrm{SD})\end{array}$ & $\begin{array}{c}2.0 \mathrm{~g}+ \\
8.0 \mathrm{~g}+ \\
50 \mathrm{~g}+ \\
50 \mathrm{ml}\end{array}$ & $\begin{array}{c}98.52 \\
(84.27)\end{array}$ & 70.68 & $\begin{array}{c}04.45 \\
(09.97)\end{array}$ & $\begin{array}{c}04.60 \\
(10.15)\end{array}$ & $\begin{array}{c}04.16 \\
(11.45)\end{array}$ & $\begin{array}{c}96.64 \\
(83.26)\end{array}$ & $\begin{array}{c}94.06 \\
(78.41)\end{array}$ & $\begin{array}{c}95.35 \\
(79.56)\end{array}$ & 91.11 \\
\hline $\mathrm{T}_{14}$ & $\begin{array}{c}\text { Carbendazim } 50 \\
\text { WP }(\mathrm{ST})+T . \\
\text { harzianum }(\mathrm{ST})+ \\
\text { Cotton seed cake } \\
\text { (SA) + } \\
\text { Z.officinale(SD) }\end{array}$ & $\begin{array}{c}1.0 \mathrm{~g}+ \\
8.0 \mathrm{~g}+50 \\
\mathrm{~g}+50 \mathrm{ml}\end{array}$ & $\begin{array}{c}97.78 \\
(81.40)\end{array}$ & 70.45 & $\begin{array}{c}06.67 \\
(14.96)\end{array}$ & $\begin{array}{c}07.14 \\
(15.49)\end{array}$ & $\begin{array}{c}06.91 \\
(15.23)\end{array}$ & $\begin{array}{c}96.61 \\
(82.68)\end{array}$ & $\begin{array}{c}90.68 \\
(72.20)\end{array}$ & $\begin{array}{c}93.65 \\
(75.56)\end{array}$ & 86.67 \\
\hline $\mathbf{T}_{15}$ & Control & Untreated & $\begin{array}{c}28.89 \\
(32.47)\end{array}$ & 00.00 & $\begin{array}{c}71.11 \\
(57.49)\end{array}$ & $\begin{array}{c}76.67 \\
(61.12)\end{array}$ & $\begin{array}{c}73.52 \\
(59.01)\end{array}$ & $\begin{array}{c}00.00 \\
(00.00)\end{array}$ & $\begin{array}{c}00.00 \\
(00.00)\end{array}$ & $\begin{array}{c}00.00 \\
(00.00)\end{array}$ & 06.67 \\
\hline & S.E. \pm & -- & 1.96 & -- & 1.72 & 2.12 & 1.92 & 2.73 & 2.74 & 1.93 & -- \\
\hline & C.D. $(P=0.05)$ & -- & 5.687 & -- & 5.00 & 6.16 & 5.58 & 7.91 & 7.96 & 5.60 & -- \\
\hline
\end{tabular}


Similar trend in respect of the post- emergence seedling mortality (PESM) was also observed and it was ranged from 4.60 to 36.67 per cent, as against 76.67 per cent in untreated control (sick soil). Of the treatments, Carbendazim $12 \%+$ Mancozeb $63 \%$ + Trichoderma viride + Neem seed cake $+A$. indica and Carbendazim $50 \mathrm{WP}+T$. harzianum + Cotton seed cake $+Z$. officinale were found most effective with significantly least post-emergence seedling mortality of 4.60 and 7.14 per cent respectively and both of which were at par.

These followed by the treatments viz., Carbendazim 12\% + Mancozeb 63\% (9.52\%), Carbendazim 50 WP (12.45\%), Thiram 37.5\% + Carboxin 37.5\% (15.38 \%), Trichoderma viride (18.38\%), T. harzianum (21.58\%), Neem seed cake (22.73\%), T. hamatum (25.00 $\%)$, Cotton seed cake (26.51\%), Z. officinale (29.09\%), Groundnut cake and A. indica (30.30 $\%)$ and $P$. hystrophorus (36.67\%).

The average mortality recorded with all the treatments was ranged from 4.16 to 35.00 per cent, as against 73.52 per cent in untreated control (sick soil). However, significantly least average mortality was recorded with Carbendazim 12\%+ Mancozeb $63 \%+$ Trichoderma viride + Neem seed cake $+A$. indica $(4.16 \%)$.

This was followed by the treatments viz., Carbendazim $50 \mathrm{WP}+T$. harzianum + Cotton seed cake + Z.officinale $(6.91 \%)$, Carbendazim $12 \%$ + Mancozeb 63\% (8.56 \%), Carbendazim 50 WP (11.41 \%), Thiram 37.5\% + Carboxin $37.5 \%$ (14.36 \%), Trichoderma viride (17.34 $\%)$, T. harzianum (20.42\%), Neem seed cake (21.73\%), T. hamatum (22.50\%), Cotton seed cake $(25.11 \%)$, Groundnut cake and A. indica (28.49 \%), Z. officinale (30.84 \%), and $P$. hystrophorus $(35.00 \%)$.

\section{Reduction in mortalities (PESR and PESM)}

All the treatments were found to reduce / control both the mortalities (pre- and postemergence) over untreated control (Table 1).
The percentage reduction in pre-emergence and post-emergence mortalities was ranged from 53.04 to 96.64 per cent and 51.95 to 94.06 per cent, respectively.

Of the treatments significantly highest reduction in PESR (96.64 \%) and PESM (94.06 \%) were recorded with Carbendazim 12\%+ Mancozeb $63 \%+$ Trichoderma viride + Neem seed cake + A. indica.

The second and third best treatments found were The second and third best treatments found were Carbendazim 50 WP $+T$. harzianum + Cotton seed cake + Z.officinale and Carbendazim 12\% + Mancozeb 63\% with second and third highest reduction in PESR (96.61 and $90.68 \%$ ) and (90.58 and $87.26 \%$ ), respectively.

These were followed by the treatments viz., Carbendazim 50 WP (84.55 and $83.79 \%$ ), Thiram 37.5\% + Carboxin 37.5\% (81.22 and $79.92 \%)$, Trichoderma viride (78.19 and 76.14 $\%)$, T. harzianum (74.85 and $71.92 \%), T$. hamatum (71.82 and $67.36 \%$ ), Neem seed cake (68.79 and $70.39 \%)$, Cotton seed cake (65.75 and $65.34 \%$ ), Groundnut cake (62.42 and 60.61 $\%)$, A. indica (62.42 and $60.36 \%), Z$. officinale (56.06 and $62.51 \%$ ), and $P$. hystrophorus (53.04 and $51.95 \%)$.

The average reduction in mortality (PESR and PESM) recorded with all the treatments were ranged 52.49 (P.hystrophorus) to 95.35 (Carbendazim 12\%+ Mancozeb $63 \%+$ Trichoderma viride + Neem seed cake $+A$. indica) per cent over untreated control. However, significantly highest reduction in average mortality $(95.35 \%)$ was recorded with the treatment Carbendazim 12\%+ Mancozeb $63 \%+$ Trichoderma viride + Neem seed cake + A. indica. This was followed by the treatments viz., Carbendazim $50 \mathrm{WP}+T$. harzianum + Cotton seed cake + Z.officinale (93.65\%), Carbendazim 12\% + Mancozeb 63\% (90.25\%), Carbendazim 50 WP (84.17\%), Thiram 37.5\% + Carboxin 37.5\% (80.57 \%), Trichoderma viride $(77.16 \%), T$. harzianum (73.39\%), T. 
hamatum and Neem seed cake (69.59 \%), Cotton seed cake $(65.55 \%)$, Groundnut cake (61.52 \%), A. indica (61.39\%), Z.officinale (56.84\%), and P. hystrophorus (52.49\%).

Similar studies were carried out by Haque et al., (2001) and Dandnaik et al., (2006) they studied the integrated control of brinjal and groundnut against foot rot / collar rot / stem rot disease.

\section{Final plant stand}

It was also revealed that all the treatments imposed were found effective against $M$. phaseolina, causing stem / root rot of brinjal and encouraged better final plant standing the range of 42.22 to 91.11 per cent, as against 6.67 per cent in untreated control.

However, highest percentage of final plant stand was recorded with the treatment Carbendazim 12\%+ Mancozeb $63 \%+$ Trichoderma viride + Neem seed cake $+A$. indica $(91.11 \%)$. This was followed by the treatments viz., This was followed by the treatments viz., Carbendazim $50 \mathrm{WP}+T$. harzianum + Cotton seed cake + Z.officinale (86.67 \%), Carbendazim 12\% + Mancozeb 63\% (82.22 \%), Carbendazim 50 WP (77.78 \%), Thiram 37.5\% + Carboxin 37.5\% (73.33\%), Trichoderma viride (68.89 \%), T. harzianum (66.67\%), T. hamatum and Neem seed cake (60.00 \%), Cotton seed cake (55.56\%), Groundnut cake and A. indica (51.11\%), Z.officinale (48.89\%), and $P$. hystrophorus $(42.22 \%)$.

It is concluded that all the treatments significantly improved the percentage seed germination over untreated control (sick soil). As well as all the treatments reduces or controls both mortalities i.e., pre- and post-emergence, over untreated control Treatment Carbendazim $12 \%+$ Mancozeb $63 \%$ + Trichoderma viride + Neem seed cake $+A$. indica were found to be most significant with highest germination and reduction in PESR (96.64 \%) and PESM (94.06 $\%)$.

\section{References}

Dandnaik, B. P., Dhoke, P. K. and Pensalwar, S. N. 2006.Integrated management of stem rot (Sclerotium rolfsii) in groundnut. Journal of Plant Disease Sciences. 1(2): 234-235.

Dinkaran, D., Sachithanantham, K. and Nainamohammed, S. E. 2005.Screening of sesame genotypes against root rot pathogen, Macrophomina phaseolina (Tassi.)Goid. Indian Journal of Plant Pathology. 23 (1\&2):66-68.

Haque, M. M.; Siddique, M. B. Meah, M. B. and Islam, M. N. 2001. Control of foot rot of brinjal through chemicals and organic soil amendments. Journal of Biological Science. 1 (10): 946-948.

Jaiman, R. K., Jain, S. C. and Sharma, P. 2009. Field evaluation of fungicides, bioagents and soil amendments against root rot caused by Macrophomina phaseolina in cluster bean. Journal of Mycology and Plant Pathology. 39 (1): 74-76.

Konde, S. A., Raut B. T., Panzade, D. S. and Ingale S. H. 2008. Management of root/collar rot disease in soybean. Journal of Plant Disease Sciences. 3 (1): 81-83.

\section{How to cite this article:}

Dapkekar, A.G., Bholanath Mondal and Suryawanshi, A.P. 2018. Integrated Disease Management of Stem and Root Rot of Brinjal caused by Macrophomina phaseolina. Int.J.Curr.Microbiol.App.Sci. 7(09): 1914-1918. doi: https://doi.org/10.20546/ijcmas.2018.709.232 